\title{
PET concerns in bevacizumab treatment
}

\section{To the editor:}

In the recent article by Willett et al. ${ }^{1}$ on neoadjuvant therapy with bevacizumab (BV) in patients with rectal cancer, treatment effects were ascribed to therapy with the VEGF-specific antibody bevacizumab. However, because patients underwent concurrent treatment with 5-fluorouracil, we wonder if at least a portion of the treatment effects observed at 7 weeks might have been mediated by 5 -fluorouracil, either alone or through a potentially synergistic effect of the combination.

In addition, we regretted the absence of information about the marked increase in 18fluorodeoxyglucose (FGD) uptake in a projection on the cervical spine region of one patient, as shown by the PET scan 6-7 weeks after the end of neoadjuvant therapy (Fig. 1c in ref. 1). Because this finding was not observed in this patient's prior PET examinations, it does not seem appropriate to view it as a mere technical artifact; it appears highly suggestive of a distant metastasis that has grown (and consequently become visible by PET) under bevacizumab combination therapy. Unexpected tumor growth at distant sites linked to treatment targeted at the primary cancer site, such as have been observed in both animal models and cancer patients ${ }^{2}$, might be due to higher net suppression of growth-inhibiting as compared to growth-stimulating substances secreted from an individual primary tumor. The PET findings in this patient could reflect this sort of adverse effect from bevacizumab-5-fluorouracil combination therapy. Therefore, we would like the authors to comment on the apparent divergent efficacy at the site of the primary tumor as compared to distant locations.

1. Willett, C.G. et al. Nat. Med. 10, 145-147 (2004) 2. O'Reilly, M.S. et al. Cell 79, 315-328 (1994).

\section{Carsten Goessl ${ }^{1} \&$ Zarko Grozdanovic ${ }^{2}$}

${ }^{1}$ Novartis Oncology, One Health Plaza, East

Hanover, New Jersey, 07936-1080 USA Department of Radiology, ${ }^{2}$ Charité, Campus Benjamin Franklin, Free University of Berlin, Germany e-mail:carsten.goessl@pharma.novartis.com

C.G is an employee of Novartis, a pharmaceutical company that has antiangiogenic compounds in development.

\section{Willett and Jain reply:}

The objective of our study was to examine the biological and clinical effects on rectal cancer of an initial bevacizumab infusion, before the initiation of radiation therapy and 5-fluorouracil. Six patients with clinical stage T3 or T4 rectal cancer completed the neoadjuvant therapy consisting of the first bevacizumab infusion followed by three 2-week cycles of bevacizumab administered concurrently with external beam radiation therapy (50.4 Gy in 28 1.8-Gy frac-

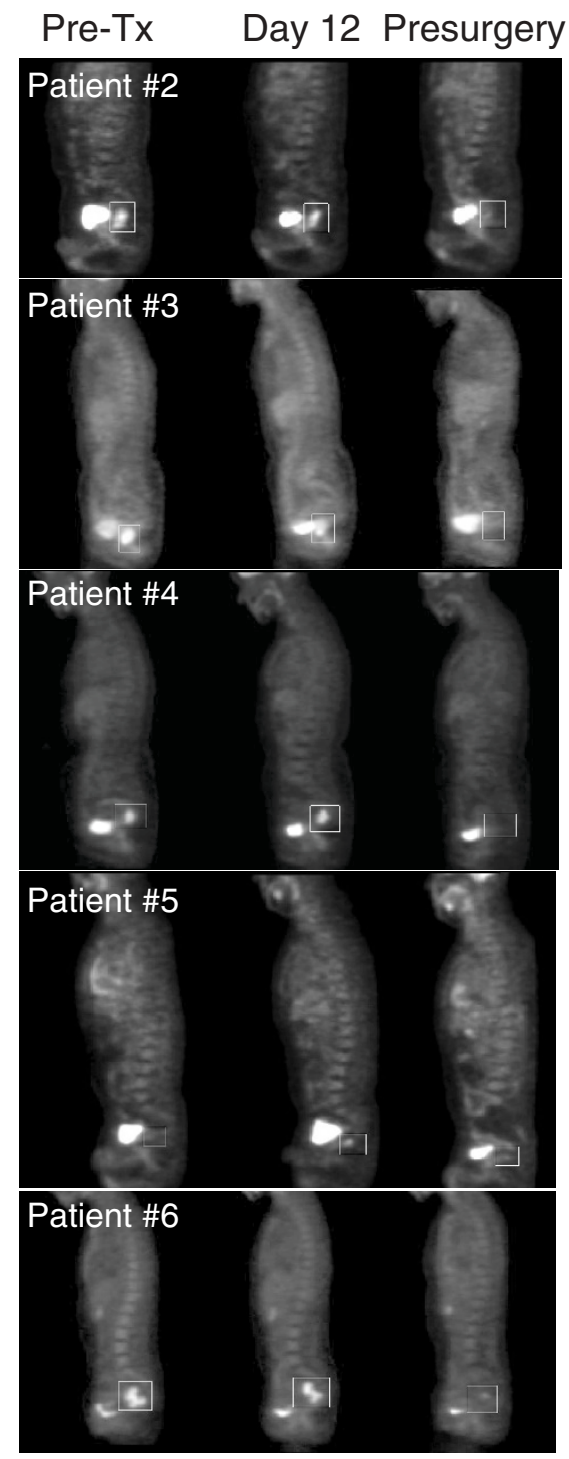

tions over 5.5 weeks) and 5-fluorouracil (225 $\mathrm{mg} / \mathrm{m}^{2} / 24 \mathrm{~h}$ by continuous peripheral venous infusion). Effects of the combined treatment on tumors were evaluated before surgery endoscopically and by PET scan and perfusion CT scan. Details of the protocol may be found in a corrigendum to our article, printed in this issue $^{1}$. The 18 -fluorodeoxyglucose uptake in the neck seen on one representative sagittal image of the presurgery PET scan (patient 1 in the original figure) was, in fact, seen on all three PET scans (pretreatment, day 12 and presurgery) and was interpreted as upper-limit normal activity in the thyroid gland. This PET finding was not judged to represent a metastasis, and similarly, PET findings showed no detectable evidence of metastasis in any of the other five patients (see Fig. 1 below). Although the goal of this study was to examine the biological and clinical response to a single dose of bevacizumab, the longer-term results of combined therapy in six consecutive patients have been promising.
Christopher G Willett ${ }^{1}$, Daniel Duda ${ }^{2}$, Alan Fischman ${ }^{3}$ \& Rakesh K Jain ${ }^{2}$
${ }^{1}$ Department of Radiation Oncology, Duke University Medical Center, Box 3085, Durham, North Carolina 27710, USA. ${ }^{2}$ Department of Radiation Oncology, Massachusetts General Hospital and Harvard Medical School, Boston Massachusetts 02114, USA. ${ }^{3}$ Department of Radiology, Massachusetts General Hospital and Harvard Medical School, Boston, Massachusetts 02114, USA.
e-mailwillett@radonc.duke.edu
1. Willett, C.G. et al. Nat. Med. 10, 649 (2004).

Figure 1 Effect of treatment on tumors in patients who completed the entire combined treatment regimen. Tumor FDG uptake before treatment (preTx), $12 \mathrm{~d}$ after BV treatment and 6-7 weeks after completion of all neoadjuvant therapy (presurgery). Sagittal projections of FDG-PET scans for patient 2-5 are shown. Tumor is outlined in box, posterior to bladder. On day 12 after BV infusion, the follow-up PET scans showed no change in tumor FDG uptake in five patients and a $40 \%$ decrease in patient 3 . Six weeks after completion of the BV, radiation therapy and chemotherapy regimen (presurgery), follow-up PET scans showed decreased tumor FDG uptake as compared with pretreatment values in patients 1,2 , 3,4 and 6 . Tumor FDG uptake in patient 5 was low, and was similar before and at the end of therapy. 